\title{
OCULUS study: Virtual reality-based education in daily clinical practice
}

\author{
Paweł Balsam, Sonia Borodzicz, Karolina Malesa, Dominika Puchta, Agata Tymińska, \\ Krzysztof Ozierański, Łukasz Kołtowski, Michał Peller, Marcin Grabowski, \\ Krzysztof J. Filipiak, Grzegorz Opolski
}

First Chair and Department of Cardiology, Medical University of Warsaw, Poland

\begin{abstract}
Background: Atrial fibrillation $(A F)$ is associated with high risk of stroke and other thromboembolic complications. The OCULUS study aimed to evaluate the effectiveness of the three-dimensional (3D) movie in teaching patients about the consequences of $A F$ and pharmacological stroke prevention.

Methods: The study was based on a questionnaire and included 100 consecutive patients (38\% women, $62 \%$ with AF history). Using the oculus glasses and a smartphone, a 3D movie describing the risk of stroke in $A F$ was shown. Similar questions were asked immediately after, 1 week and 1 year after the projection.

Results: Before the projection 22/100 (22.0\%) declared stroke a consequence of AF, while immediately after 83/100 (83.0\%) ( $p<0.0001)$ patients declared this consequence. Seven days after, stroke as AF consequence was chosen by 74/94 (78.7\%) vs. 22/94 (23.4\%) when compared to the baseline knowledge; $p<0.0001$, a similar trend was also observed in 1-year follow-up (64/90 [71.1\%] vs. 21/90 [23.3\%]; $p<0.0001$ ). Before the projection $88.3 \%$ (83/94) patients responded, that drugs may reduce the risk of stroke, and after 1 week the number of patients increased to $(94 / 94$ [100\%]; $p=0.001)$. After 1 year $87 / 90$ (96.7\%) answered that drugs may diminish the risk of stroke ( $p=0.02 \mathrm{in}$ comparison to the baseline survey 78/90 [86.7\%]). Use of oral anticoagulation to reduce the risk of stroke was initially chosen by 66/94 (70.2\%), by 90/94 (95.7\%; $p<0.0001) 7$ days after and by $83 / 90(92.2 \% ; p<0.0001)$ 1 year after.

Conclusions: $3 D$ movie is an effective tool in transferring knowledge about the consequences of $A F$ and the pivotal role of oral anticoagulation in stroke prevention. (Cardiol J 2019; 26, 3: 260-264)

Trial registration: ClinicalTrials.gov, NCT03104231. Registered on 28 March 2017.

Key words: atrial fibrillation, stroke, education, virtual reality, three-dimensional movie
\end{abstract}

\section{Introduction}

Atrial fibrillation (AF) is the most common cardiac arrhythmia in adults, with a significant risk of morbidity and mortality, mainly due to increased risk of stroke and systemic embolism. AF is independently correlated with a 2 -fold increased risk of all-cause mortality in women and a 1.5-fold in men [1]. The prevalence of AF is approximately $3 \%$ in adults aged 20 years or older and increases with age and presence of hypertension, heart failure, coronary artery disease, valvular heart disease, obesity, diabetes mellitus and chronic kidney disease [1].

Nowadays, identification and prevention of AF risk factors, as well as prevention of thromboembolic events constitute the fundamentals of comprehensive care in AF [1]. Most thromboembolic events may be prevented by oral anticoagulation $(\mathrm{OAC})$, which is suggested to be prescribed to the majority of patients with $\mathrm{AF}$ basing on $\mathrm{CHA}_{2} \mathrm{DS}_{2}$ -

Address for correspondence: Sonia Borodzicz, $1^{\text {st }}$ Chair and Department of Cardiology, Medical University of Warsaw, Public Central Teaching Hospital in Warsaw, ul. Banacha 1a, 02-097 Warszawa, Poland, tel: +48 22 5991958, fax: +48 22 5991957, e-mail: sonia.borodzicz@onet.pl 
-VASc score [1]. The current guidelines for the management of AF patients highlight the important role of education in treatment $[1,2]$. Therefore, one of the most important factors leading to the achievement of clinical benefit from OAC therapy is an effective cooperation of patients with physicians. Thus, physicians should introduce novel e-solutions to the communication process with patients to increase compliance [3]. Three-dimensional (3D) and virtual reality (VR) models are helpful in every day medical practice $[4,5]$ but could be also used as educational tools.

The OCULUS study aimed to evaluate whether the VR-3D movie-based education is effective in improving patient knowledge about the consequences of $\mathrm{AF}$ and pharmacological possibilities in reducing the risk of stroke. An additional objective of the analysis was to assess factors contributing to OAC usage in the prevention of stroke.

\section{Methods}

\section{Study design and population}

The OCULUS was a prospective, single center study. The study included hospitalized patients, who were over 18 years old. Only patients with previously diagnosed dementia were excluded from the study. The recruitment phase of the OCULUS study lasted from April 2016 to August 2016. Local ethics committees approved the study. All patients were provided with detailed information and signed informed written consent.

The study was based on a questionnaire (Suppl. material), designed by the authors of the study. The questionnaire was composed of questions about sex, age, education, current job, AF history, as well as knowledge about consequences of $\mathrm{AF}$ and the importance of OAC therapy in stroke prevention. Consequently, the brief VR-3D movie which is available on Google Play and Appstore was shown using oculus glasses and a smartphone (a version prepared for men is available at the address: https://www.youtube.com/ watch? $v=5 \mathrm{WFxq} m 88 \mathrm{ds}$, whereas a version for women is available at the address: https://www. youtube.com/watch? $v=$ S8i7LxBBv0g). The Pfizer Company allowed the authors of the study to use the movie. The movie's plot to inform patients about the risk of stroke and possibilities of its prevention by using OAC. Subsequently, patients were asked a few questions, including what the movie was about, whether AF can affect a patient's life in a negative way, whether it is possible to reduce the risk of stroke and if or by what types of drugs also reduce the risk of stroke. Similar questions were asked to the patients immediately after the movie, 1 week and 1 year later (via telephone follow-up).

\section{Statistical analysis}

Categorical variables are presented as a percentage of total for the group. Comparisons of results before and after watching the movie was made using the McNemar test. Bonferroni correction was used for multiple comparisons. Logistic regression was performed to assess factors contributing to OAC usage as a prevention of stroke. A value of $\mathrm{p}<0.05$ was considered significant. The calculations were performed using SAS software 9.4.

\section{Results}

The current analysis included 100 patients (38\% women). Mean age of the study group was $63 \pm 15$ years. Data on 7-day follow-up were available for 94 patients ( 6 patients were lost to telephone follow-up), whereas the 1 -year follow-up data were collected from 90 patients (10 patients had not completed the telephone follow-up). Previous history of AF was reported in $62 / 100(62.0 \%)$ of the patients examined. Before projection of the movie $22 / 100(22.0 \%)$ of patients answered that stroke is a dangerous consequence of $\mathrm{AF}$, while immediately after the projection the number of patients significantly increased (83/100 [83.0\%]; $p<0.0001)$. One week after the number of patients choosing stroke as a consequence of $\mathrm{AF}$ was still significantly higher in comparison to the knowledge before the projection (74/94 [78.7\%] vs. 22/94 [23.4\%]; $\mathrm{p}<0.0001)$, similar trend was observed also in the 1-year follow-up (64/90 [71.1\%] vs. 21/90 [23.3\%]; $\mathrm{p}<0.0001$ ) (Fig. 1). High number of patients $(83 / 94 ; 88.3 \%)$ asked prior to the projection and all patients $(94 / 94 ; 100 \%)$ who were asked after 7 days responded that the risk of stroke may be reduced by using a specific pharmacological therapy $(\mathrm{p}=0.001)$. Moreover, in 1-year follow-up 87/90 $(96.67 \%)$ of patients confirmed that specific drugs may serve as a possibility to diminish stroke risk ( $p=0.023$ in comparison to the survey before the projection $78 / 90[(86.7 \%])$. As for drugs reducing the risk of stroke, OAC chosen by 66/94 (70.2\%) of patients prior to the projection and by $90 / 94$ $(95.7 \%)$ of patients a week after watching the movie $(\mathrm{p}<0.0001)$. Importantly, 1 year following the projection the knowledge of $\mathrm{OAC}$ as a pharmacologic possibility of stroke risk reduction was significantly improved in comparison to the knowledge prior watching the movie (83/90 [92.2\%] vs. 


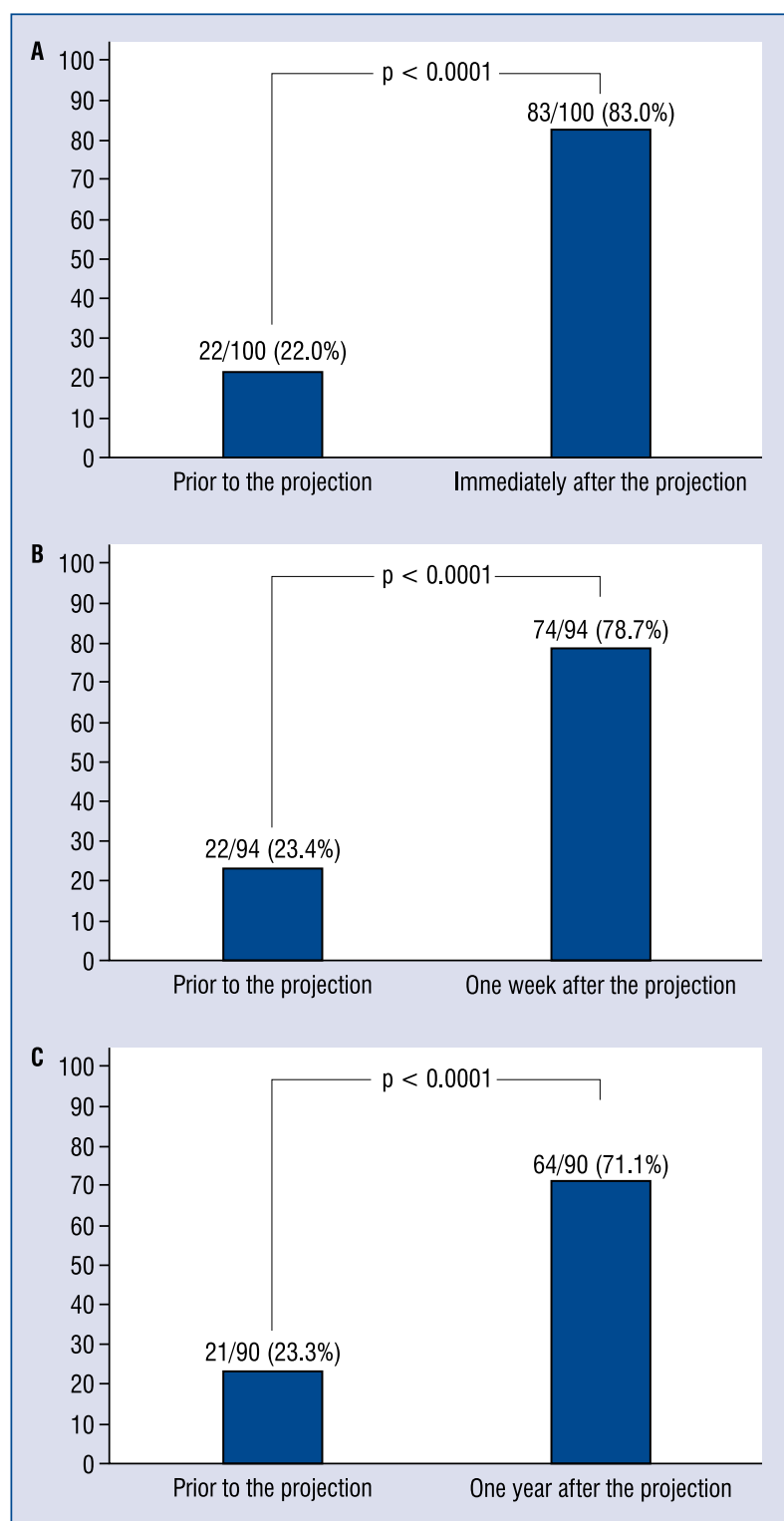

Figure 1. Percentages of patients declaring stroke a dangerous consequence of atrial fibrillation; A. Immediately after the projection; B. One week after the projection; C. One year after the projection.

60/90 [66.7\%]; p < 0.0001) (Fig. 2). Before the projection 54/94 (57.5\%) of patients confirmed previous treatment with $\mathrm{OAC}$, whereas 7 days after the projection the usage of OAC was declared by $66 / 94(70.2 \%)$ of the patients asked $(\mathrm{p}=0.004)$. Also, 1 year after the projection significantly higher number of patients confirmed use of OAC therapy when compared to the baseline survey $(65 / 90$ [72.2\%] vs. 50/90 [55.6\%]; $p=0.006$ ) (Fig. 3). The VR-3D movie was acknowledged as a useful tool to spread awareness of consequences of AF by $99 / 100$ (99.0\%) of the study participants. Approximately

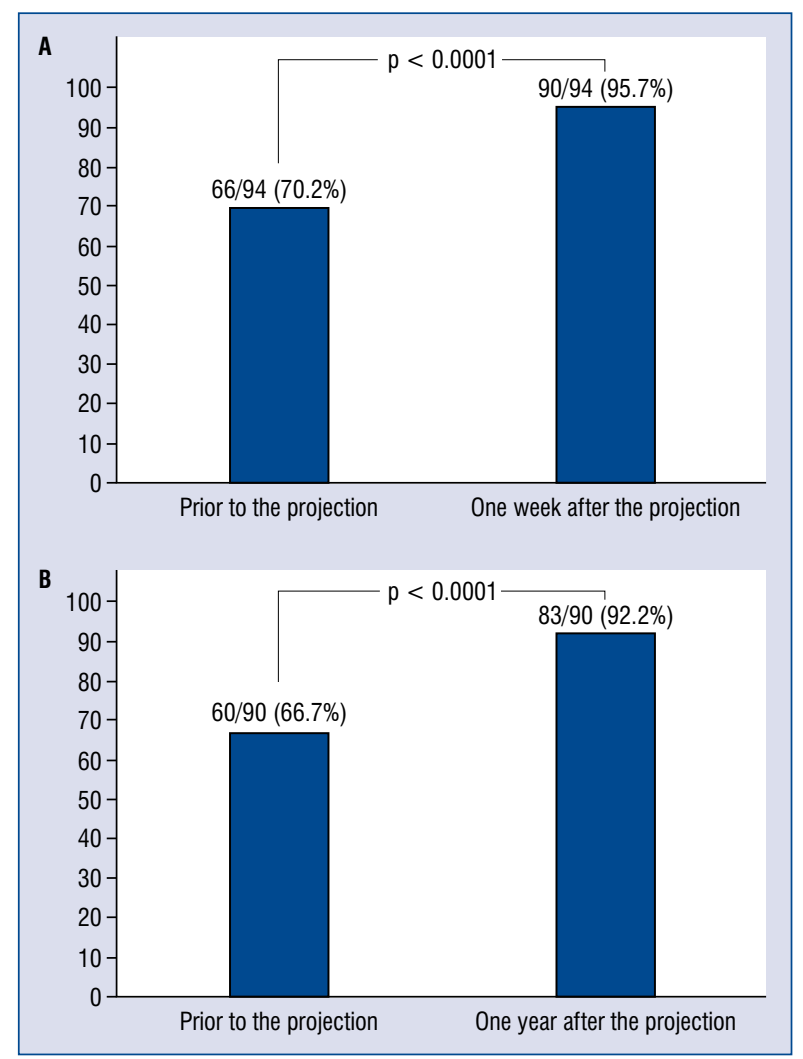

Figure 2. Percentages of patients choosing oral anticoagulants as drugs which reduce the risk of stroke; A. One week after projection; B. One year after projection.

all of the patients $(99 / 100 ; 99.0 \%)$ confirmed that they would enjoy watching similar movies about other diseases. One week after watching the movie $91 / 93$ (97.9\%) of patients declared using prescribed OAC therapy in the future, due to the gained knowledge through watching the movie about the consequences associated with $\mathrm{AF}$. The univariate analysis did not reveal any statistically significant relations between age or gender, and the frequency of marking stroke as a consequence of $\mathrm{AF}$ or choosing $\mathrm{OAC}$ as a possibility of reducing the risk of stroke.

\section{Discussion}

The results obtained clearly show that knowledge about the consequences of $\mathrm{AF}$ results in an improved declared adherence to treatment with OAC. The vast majority of patients acknowledged that the VR-3D movie technology is an interesting and useful tool to spread the awareness about AF, as well as other diseases.

Results from the European Heart Rhythm Association (EHRA) Survey conducted in 53 


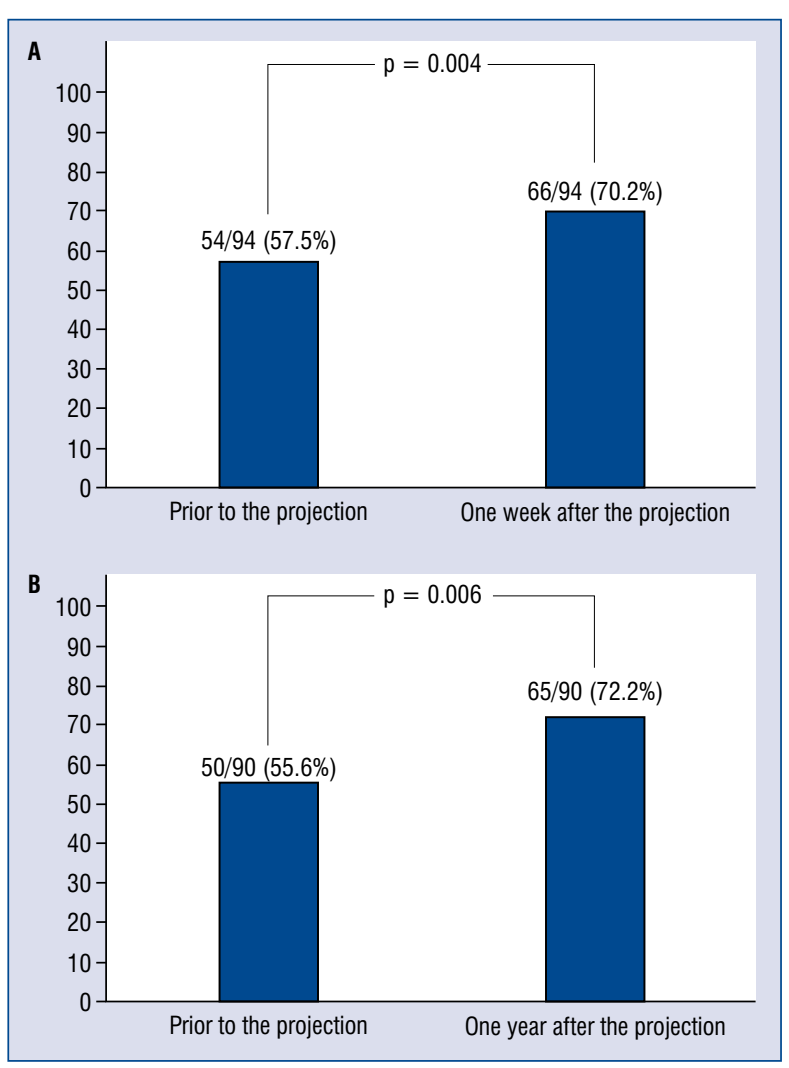

Figure 3. Percentages of patients who declare taking oral anticoagulants previously; A. One week after projection; B. One year after projection.

European centres revealed, that stroke and bleeding risk management had the highest priority in discussion with AF patients in majority of centres (80.2\%) [6]. However, none of the centres used the mobile phone applications to explain the risk of stroke, as a consequence of AF. The majority of centres $(92.3 \%)$ included in this survey provided discussion with patients to describe the risk of stroke in AF. In addition to the conversation, some of the centres provided illustrative materials from pharmaceutical companies (23.1\%), other printed materials (17.3\%), links to websites (17.3\%), specialized nurse $(11.5 \%)$ or health psychologist (1 center). Moreover, there was estimated that the percentages of patients who would desist OAC despite the knowledge of advantages and risks of the therapy were: $0.0 \%$ in $13.7 \%$ of the centers, $\leq 10 \%$ in $58.8 \%, 11-20 \%$ in $21.6 \%$ and $21-30 \%$ in $5.9 \%$ of the centers. Interestingly, the prevailing reasons for OAC withdrawal were patient fear of bleeding ( $41.2 \%$ of the centers), underestimation of stroke risk despite having the proper knowledge (21.6\%), deficient patient information about the risk of stroke (15.7\%), unknown or other reasons
(21.6\%) [6]. Another EHRA Survey evaluating the level of education about the OAC, conducted in 8 European centres reported, that $90 \%$ of patients with $\mathrm{AF}$ give the proper explanation for the $\mathrm{OAC}$ therapy (which was said as "to thin the blood") [7].

It is known that management of OAC is challenging because of the need for an appropriate balance between hemorrhagic and thromboembolic risks, as well as due to different phenotypes of patients using this therapy. Interestingly, results from the EHRA Survey show that patients without schooling had the highest percentage of bleeding on OAC and previous strokes and the lowest use of OAC for stroke prevention [2]. The OCULUS study did not show any statistically significant correlations between age or gender, and the frequency of marking stroke as a consequence of $\mathrm{AF}$ or choosing $\mathrm{OAC}$ as a possibility in reducing the risk of stroke.

In 2014 Cleeren et al. [8] conducted a randomized controlled trial which evaluated gaining and recall of knowledge on periodontitis by patients suffering from periodontitis. The authors revealed, that patients who were shown 3D animations had significantly higher scores in knowledge immediately after the projection and in the following 2 weeks in comparison to a narration and drawing group. Therefore, authors of the study agreed, that $3 \mathrm{D}$ animations may be a useful tool in the education of patients. VR is also suggested to be effective as a diagnostic application in detecting mild cognitive impairment [9]. Moreover, VR is concerned to be a useful additional tool in rehabilitation after ischemic stroke and cerebral palsy [10, 11]. It has been proven, that patients using VR applications experience less pain and distress, furthermore they also declare awillingness to use VR during the medical procedures related with pain [12]. Also other educational tools, such as an iPad-based application (iBook), is suggested to be beneficial in otologic diseases in improving patient knowledge and satisfaction [13].

\section{Limitations of the study}

Some important limitations of the OCULUS study have to be acknowledged. First of all, the analyzed group of patients is relatively small. Secondly, a longer follow-up is required to assess the effectiveness of the 3D movie on patient knowledge and attitude towards compliance. Moreover, the OCULUS study enrolled only hospitalized patients. However, it could be hypothesized that, those patients may have a higher level of knowledge about $\mathrm{AF}$ and anticoagulation treatment and what may have had a significant influence on the obtained 
results. Thus, there is a need to conduct such a study in the general population.

\section{Conclusions}

The OCULUS study shows, that a 3D movie is an effective tool in teaching patients about the consequences of $\mathrm{AF}$ and the important role of $\mathrm{OAC}$ in stroke prevention. Due to the interesting results of the current study, there is a need to create educational VR-3D movies, which may contribute to the improvement in patient compliance and better treatment outcomes for other diseases. Further multicentre studies would be helpful to evaluate the role of VR-3D-movie-based knowledge transfer for several diseases, not only in $\mathrm{AF}$.

\section{Trial registration}

The study has been registered in the ClinicalTrials.gov, NCT03104231. Registered on 28 March 2017.

\section{Conflict of interest: None declared}

\section{References}

1. Kirchhof P, Benussi S, Kotecha D, et al. 2016 ESC Guidelines for the management of atrial fibrillation developed in collaboration with EACTS. Rev Esp Cardiol. 2017; 70(1): 50, doi: 10.1016/j. rec.2016.11.033, indexed in Pubmed: 28038729.

2. Hernández Madrid A, Potpara TS, Dagres N, et al. Differences in attitude, education, and knowledge about oral anticoagulation therapy among patients with atrial fibrillation in Europe: result of a self-assessment patient survey conducted by the European Heart Rhythm Association. Europace. 2016; 18(3): 463-467, doi: 10.1093/europace/euv448, indexed in Pubmed: 26899998.

3. Milewski K, Małecki A, Orszulik-Baron D, et al. The use of modern telemedicine technologies in an innovative optimal cardiac rehabilitation program for patients after myocardial revascularization: Concept and design of RESTORE, a randomized clinical trial. Cardiol J. 2018 [Epub ahead of print], doi: 10.5603/ /CJ.a2018.0157, indexed in Pubmed: 30566211.

4. Rymuza B, Grodecki K, Kamiński J, et al. Wykorzystanie hologramu podczas przezcewnikowego wszczepienia zastawki aor- talnej u pacjenta ze stenozą dwupłatkowej zastawki aortalnej. Kardiol Pol. 2017; 75(10): 1056-1056, doi: 10.5603/kp.2017.0195, indexed in Pubmed: 29057443.

5. Olejník P, Nosal M, Havran T, et al. Utilisation of three-dimensional printed heart models for operative planning of complex congenital heart defects. Kardiol Pol. 2017; 75(5): 495-501, doi: 10.5603/KP.a2017.0033, indexed in Pubmed: 28281732.

6. Pison L, Potpara TS, Chen J, et al. Scientific Initiative Committee, European Heart Rhythm Association, Scientific Initiatives Committee, and European Heart Rhythm Association. How are patients with atrial fibrillation approached and informed about their risk profile and available therapies in Europe? Results of the European Heart Rhythm Association Survey. Europace. 2015; 17(3): 468-472, doi: 10.1093/europace/euv025, indexed in Pubmed: 25722478.

7. Amara W, Larsen TB, Sciaraffia E, et al. Patients' attitude and knowledge about oral anticoagulation therapy: results of a selfassessment survey in patients with atrial fibrillation conducted by the European Heart Rhythm Association. Europace. 2016; 18(1): 151-155, doi: 10.1093/europace/euv317, indexed in Pubmed: 26462697.

8. Cleeren G, Quirynen M, Ozcelik O, et al. Role of 3D animation in periodontal patient education: a randomized controlled trial. J Clin Periodontol. 2014; 41(1): 38-45, doi: 10.1111/jcpe.12170, indexed in Pubmed: 24111854.

9. Zygouris S, Ntovas K, Giakoumis D, et al. A Preliminary Study on the Feasibility of Using a Virtual Reality Cognitive Training Application for Remote Detection of Mild Cognitive Impairment. J Alzheimers Dis. 2017; 56(2): 619-627, doi: 10.3233/ /JAD-160518, indexed in Pubmed: 28035922.

10. Schmid L, Glässel A, Schuster-Amft C. Therapists' Perspective on Virtual Reality Training in Patients after Stroke: A Qualitative Study Reporting Focus Group Results from Three Hospitals. Stroke Res Treat. 2016; 2016: 6210508, doi: 10.1155/2016/6210508, indexed in Pubmed: 28058130.

11. Ravi DK, Kumar N, Singhi P. Effectiveness of virtual reality rehabilitation for children and adolescents with cerebral palsy: an updated evidence-based systematic review. Physiotherapy. 2017; 103(3): 245-258, doi: 10.1016/j.physio.2016.08.004, indexed in Pubmed: 28109566.

12. Li A, Montaño Z, Chen VJ, et al. Virtual reality and pain management: current trends and future directions. Pain Manag. 2011; 1(2): 147-157, doi: 10.2217/pmt.10.15, indexed in Pubmed: 21779307.

13. Moshtaghi O, Haidar YM, Sahyouni R, et al. Use of interactive iBooks for patient education in otology. Am J Otolaryngol. 2017; 38(2): 174-178, doi: 10.1016/j.amjoto.2016.12.004, indexed in Pubmed: 28089173. 